\title{
DIFFERENTIAL EQUATIONS CONTAINING ARBITRARY
}

\section{FUNCTIONS*}

\author{
BY \\ GILBERT AMES BLISS
}

A recent study of the problem of computing differential corrections for a trajectory has led the writer to the present investigation of the properties of solutions of differential equations considered as functions of other arbitrary functions which enter into the differential equations themselves. In ballistics it is customary to compute first the coördinates of a trajectory, uninfluenced by wind or other disturbances, as solutions of the differential equations of motion. A new system of differential equations is then set up which accounts for the disturbances of the trajectory, and with the help of which corrections to the coördinates of the projectile on the original trajectory can be computed. The new equations involve arbitrary functions and the corrections found are themselves functions of these functions. The details of this situation will be described in another paper. In the following pages the results attained are of a more general character than is necessary for the ballistic problem, and it seems probable that they will be useful in many other connections.

The differential equations here considered have the form

$$
\frac{d x^{(i)}}{d \tau}=f^{(i)}\left(\tau, x^{(1)}, \cdots, x^{(p)}\right) . \quad(i=1,2, \cdots, p),
$$

and it is supposed that a particular solution

$$
x^{(i)}=u^{(i)}(\tau) \quad\left(i=1, \cdots ; p ; \tau_{1} \leqq \tau \leqq \tau_{2}\right)
$$

for a particular set of functions $f^{(i)}$ is known. When the functions $f^{(i)}$ themselves are allowed to vary the solutions of these equations are functions $x^{(i)}\left[\tau, \tau_{0}, x_{0}, f\right]$ of the variable $\tau$, of the coördinates $\tau_{0}$ and

$$
x_{0}=\left(x_{0}^{(i)}, \cdots, x_{0}^{(p)}\right)
$$

of a prescribed initial point $\left(\tau, x_{0}\right)$ of the curve, and of the functions $f^{(i)}$ in the second members of the equations. In Sections 1 and 2 below the character of these solutions in a neighborhood of the particular solution (2) are considered.

* Presented to the Society, September, 1919. 
It is proved that they are continuous and have differentials with respect to all their arguments, and the parts of the differentials which involve the variations of the functions $f$ are differentials in a sense analogous to that of Fréchet or Volterra in the theory of functions of lines.* The variations of the functions $f$ considered in this paper are arbitrary ones. In special cases, however, they may be caused by the presence of variable parameters in the second members of the equations (1), or by the presence of arbitrary functions as is the case in ballistics.

In Section 3 some implicit function theorems are developed which have an application in the theory of the wind and density corrections in ballistics. The character of a solution $\tau=\tau\left[\tau_{0}, x_{0}, f\right]$ of an equation of the form

$$
\phi\left[\tau, \tau_{0}, x_{0}, f\right]=0
$$

is discussed. Under suitable hypotheses the solution $\tau$ will be continuous and have a differential, as will also the function obtained by substituting the solution $\tau$ in a second function $\psi$ of the same sort as $\phi$. The theorems deduced are special cases of some much more general ones in general analysis.

The notations and properties of multi-partite numbers and matrices have been used freely throughout. This gives a somewhat forbidding aspect to the pages for one who has not considered the advantages of these notations. It is a matter of a short time only, however, to familiarize one's self with their essential properties, and the instruments so provided are of very great usefulness and power in many applications. The use of multi-partite numbers and matrices in the differential equation theory was inaugurated by Peano $\dagger$ and the present writer has more recently listed in some detail the properties which are found to be most useful in this connection. The notations and methods of the paper last referred to, and which will hereafter be designated as the paper (A), will be preserved as far as possible. For example, onepartite numbers will always be represented by Greek letters, $p$-partite numbers by small Roman letters, and matrices by capital Roman letters. A list of the notations used is given on page 21 of the paper (A). It will assist in the interpretation of the following equations if it is noted that the properties of the moduli of multi-partite numbers and matrices are analogous to those of the ordinary absolute value.

\footnotetext{
* Fréchet, Sur la notion de différentielle d'une fonction de ligne, these T r a n s a c t i o n s, vol. 15 (1914), p. 139. Volterra, Leçons sur les fonctions des lignes, p. 25.

$\dagger \mathrm{M}$ a the matis che Annalen, vol. 32 (1888), p. 450.

$\ddagger$ The solutions of differential equations of the first order as functions of their initial values, Annals of Mathematics, 2d series, vol. 6 (1905), p. 58; Solutions of differential equations as functions of the constants of integration, Bulletin of the A merican M a the mat i c a 1 S o c i e t y, 2d series, vol. 24 (1918), p. 21.
} 


\section{Solutions of differential Equations containing arbitrary FUNCTIONS}

The system (1) of differential equations is represented in matrix notation by the single equation

$$
\frac{d x}{d \tau}=f(\tau, x)
$$

Interpreted from the standpoint of multi-partite numbers the latter means that a similar equation holds for each value of the index $i=1,2, \cdots, p$ of equations (1). An initial solution

$$
x=u(\tau) \quad\left(\tau_{1} \leqq \tau \leqq \tau_{2}\right)
$$

of the differential equations (3) for a special set $f^{\prime}$ of functions $f$, is supposed to be known in advance, each function of the set $u$ having a continuous derivative on the interval $\tau_{1} \tau_{2}$. There is also a neighborhood $C_{\epsilon}$ of the arc $C$, consisting of all the points $(\tau, x)$ satisfying the conditions

$$
\tau_{1} \leqq \tau \leqq \tau_{2}, \quad \bmod [x-u(\tau)]<\epsilon,
$$

in which all the functions $f$ to be considered are single valued and continuous and satisfy the Lipschitz conditions

$$
\bmod \left[f\left(\tau, x^{\prime}\right)-f(\tau, x)\right]<\kappa \bmod \left(x^{\prime}-x\right)
$$

with a prescribed constant $\kappa$ which is the same for all the $f$ 's considered. This would, for example, be the case for all functions $f$ having suitably bounded continuous first derivatives in $C_{\mathrm{e}}$.

Under the circumstances described in the preceding paragraph a sequence of sets of approximation functions $v_{m}=\left(v_{m}^{(1)}, \cdots, v_{n}^{(p)}\right)$ may be defined by the equations

$$
\begin{aligned}
v_{0}\left[\tau, \tau_{0}, x_{0}, f\right] & =u(\tau)+x_{0}-u\left(\tau_{0}\right), \\
v_{m+1}\left[\tau, \tau_{0}, x_{0}, f\right] & =x_{0}+\int_{\tau_{0}}^{\tau_{0}} f\left(\tau, v_{m}\right) d \tau,
\end{aligned}
$$

and each set of functions $v_{m}$ has the initial values $x_{0}$ when $\tau=\tau_{0}$.

To justify the use of these formulas it must first be shown, as in the paper (A), that there is a domain $R$ of elements $\left[\tau, \tau_{0}, x_{0}, f\right]$ in which every one of the sets $v_{m}$ is well defined and provides only points $\left(\tau, v_{m}\right)$ in the region $C_{\text {. }}$ where the continuity properties of the functions $f$ have been presupposed. Such a domain $R$ is defined by the conditions

$$
\tau_{1} \leqq \tau \leqq \tau_{2}, \quad\left(\tau_{0}, x_{0}\right) \text { in } C_{\delta / 2}, \quad \bmod \left(f-f^{\prime}\right) \leqq \frac{\delta}{2 \lambda},
$$

where $\lambda$ is the length of the interval $\tau_{1} \tau_{2}$ and $\delta=\epsilon e^{-\kappa \lambda}$. For the functions $u$ clearly satisfy the equations 


$$
u(\tau)=u\left(\tau_{0}\right)+\int_{\tau_{0}}^{\tau} f^{\prime}(\tau, u) d \tau
$$

and from equations (5), (4), and properties of the modulus analogous to those of the absolute value, it follows that in the region $R$

$$
\begin{aligned}
\bmod \left(v_{0}-u\right)= & \bmod \left[x_{0}-u\left(\tau_{0}\right)\right] \leqq \frac{\delta}{2}<\delta \\
\bmod \left(v_{1}-u\right) \leqq & \bmod \left[x_{0}-u\left(\tau_{0}\right)\right]+\bmod \int_{\tau_{0}}^{\tau}\left[f\left(\tau, v_{0}\right)-f^{\prime}(\tau, u)\right] d \tau \\
\leqq & \frac{\delta}{2}+\left|\int_{\tau_{0}}^{\tau} \bmod \left[f\left(\tau, v_{0}\right)-f^{\prime}\left(\tau, v_{0}\right)\right] d \tau\right| \\
& +\left|\int_{\tau_{0}}^{\tau} \bmod \left[f^{\prime}\left(\tau, v_{0}\right)-f^{\prime}(\tau, u)\right] d \tau\right| \\
\leqq & +\left|\int_{\tau_{0}}^{\tau} \kappa \bmod \left(v_{0}-u\right) d \tau\right| \\
\leqq & \left\{1+\frac{\kappa\left|\tau-\tau_{0}\right|}{1 !}\right\} .
\end{aligned}
$$

A simple inductive proof now gives

$$
\bmod \left(v_{m}-u\right) \leqq \delta\left\{1+\frac{\kappa\left|\tau-\tau_{0}\right|}{1 !}+\cdots+\frac{\kappa^{m}\left|\tau-\tau_{0}\right|^{m}}{m !}\right\} \leqq \delta e^{\kappa \lambda}=\epsilon,
$$

which shows that the points $\left(\tau, v_{m}\right)$ are in $C_{e}$ for every $m$ and every set of arguments $\left[\tau, \tau_{0}, x_{0}, f\right]$ in $R$.

The proofs (1) that the sequence of functionals $v_{m}\left[\tau, \tau_{0}, x_{0}, f\right]$ converges uniformly in the domain $R$ to a limiting set $v\left[\tau, \tau_{0}, x_{0}, f\right]$, (2) that this limiting set satisfies the equations

$$
v\left[\tau, \tau_{0}, x_{0}, f\right]=x_{0}+\int_{\tau_{0}}^{\tau} f(\tau, v) d \tau
$$

and hence also the original equations (3), and (3) that when $\tau_{0}, x_{0}, f$ are prescribed the functions $v$ are the only ones which satisfy equations (3), are now precisely those of the paper (A) cited above, and they need not be reproduced here.

The approximation functionals $v_{m}\left[\tau, \tau_{0}, x_{0}, f\right]$ have uniform continuity in the domain $R$ of the following type, designated in the rest of this paper as continuity of type I. Let $\phi$ be one of them, and designate by $\Delta \phi$ the difference

$$
\Delta \phi=\phi\left[\tau+\Delta \tau, \tau_{0}+\Delta \tau_{0}, x_{0}+\Delta x_{0}, f+\Delta f\right]-\phi\left[\tau, \tau_{0}, x_{0}, f\right] .
$$

Then for every positive constant $\epsilon^{\prime}$ there is a second constant $\delta^{\prime}$ such that 
$|\Delta \phi|<\epsilon^{\prime}$ whenever the sets $\left[\tau+\Delta \tau, \tau_{0}+\Delta \tau_{0}, x_{0}+\Delta x_{0}, f+\Delta f\right]$ and $\left[\tau, \tau_{0}, x_{0}, f\right]$ are in $R$ and

$$
|\Delta \tau|,\left|\Delta \tau_{0}\right|, \bmod \Delta x_{0}, \bmod \Delta f<\delta^{\prime} .
$$

The last inequality is supposed to hold uniformly for all points $(\tau, x)$ in $C_{\mathrm{e}}$.

The proof of this property of uniform continuity is an inductive one. The function $v_{0}$ is evidently continuous in the sense just described, and it is assumed for purposes of proof that $v_{m}$ has the same property. It follows then from equations (5) that

$$
\begin{aligned}
& \Delta v_{m+1}=\Delta x_{0}+\int_{\tau_{0}+\Delta \tau_{0}}^{\tau+\Delta \tau} \bar{f}\left(\tau, v_{m}+\Delta v_{m}\right) d \tau-\int_{\tau_{0}}^{\tau} f\left(\tau, v_{m}\right) \Delta \tau \\
& =\Delta x_{0}+\int_{\tau}^{\tau+\Delta \tau} \bar{f}\left(\tau, v_{m}+\Delta v_{m}\right) d \tau+\int_{\tau_{0}+\Delta \tau_{0}}^{\tau_{0}} \bar{f}\left(\tau, v_{m}+\Delta v_{m}\right) d \tau \\
& +\int_{\tau_{0}}^{\tau} \Delta f\left(\tau, v_{m}+\Delta v_{m}\right) d \tau+\int_{\tau_{0}}^{\tau}\left[f\left(\tau, v_{m}+\Delta v_{m}\right)-f\left(\tau, v_{m}\right)\right] d \tau,
\end{aligned}
$$

where $\bar{f}=f+\Delta f$. Hence

$\bmod \Delta v_{m+1} \leqq \bmod \Delta x_{0}+\mu\left[|\Delta \tau|+\left|\Delta \tau_{0}\right|\right]$

$$
+\left|\int_{\tau_{0}}^{\tau} \bmod \Delta f d \tau\right|+\left|\int_{\tau_{0}}^{\tau} \kappa \bmod \Delta v_{r} d \tau\right|,
$$

where $\mu$ is a maximum of $\bmod f$ in the domain $R$, and the sum of these terms can clearly be made less than $\epsilon^{\prime}$ by sufficiently restricting $\delta^{\prime}$. It follows at once from the uniform convergence of the sequence $\left\{v_{m}\right\}$ that its limit function $v\left[\tau, \tau_{0}, x_{0}, f\right]$ has also continuity of type $I$ in the domain $R$.

Suppose now that the class of sets of functions $f$ is still further restricted to include only functions which have continuous first derivatives in the neighborhood $C_{e}$; and that these derivatives have a common prescribed upper bound which is the same for all the $f$ 's considered. Then there will exist a constant $\kappa$ such that the Lipschitz condition (4) holds for all of the sets of functions. The proofs of the paper (A) repeatedly cited establish for each $f$ the existence of the derivatives of the solutions given by the equations

$$
\begin{aligned}
& \frac{\partial v}{\partial x_{0}}=I+\int_{\tau_{0}}^{\tau} f_{x} d \tau+\cdots+\int_{\tau_{0}}^{\tau} f_{x} \cdots \int_{\tau_{0}}^{\tau} f_{x} d \tau^{m}+\cdots, \\
& \frac{\partial v}{\partial \tau_{0}}=-\frac{\partial v}{\partial x_{0}} f\left(\tau_{0}, x_{0}\right) .
\end{aligned}
$$

In the former of these equations $\partial v / \partial x_{0}$ is the matrix $\left\|\partial v^{(i)} / \partial x_{0}^{(j)}\right\|(i, j=1$, $\cdots, p)$ of derivatives of the elements of $v$ with respect to the elements of $x_{0}$, 
$I$ is the identity matrix, $f_{x}$ is the matrix of derivatives of the elements of $f$ with respect to those of $x$ with the arguments $(\tau, x)=(\tau, v)$, and the equation holds for every pair of corresponding elements of the matrices on the two sides when the sums and products and integrals on the right are interpreted in the usual way. In the latter equation $\partial v / \partial \tau_{0}$ is the multi-partite number whose elements are the derivatives of the elements of $v$ with respect to the scalar $\tau_{0}$, and the second member is the negative product of the matrix $\partial v / \partial x_{0}$ by the multi-partite number $f\left(\tau_{0}, x_{0}\right)$.

The sum (6) converges uniformly in $R$, as follows at once from the proof given in the paper (A) cited above. In order therefore to show that the derivatives $\partial v / \partial x_{0}$ are uniformly continuous in $R$ it is only necessary to show that each term is uniformly continuous. The continuity to be demonstrated is, however, of a special kind which will be called continuity of type II. Let $\phi\left[\tau, \tau_{0}, x_{0}, f\right]$ be one of the derivatives. It is to be proved uniformly continuous in the sense that for every positive constant $\epsilon^{\prime}$ there is a second positive constant $\delta^{\prime}$ depending only upon $f$ and $\epsilon^{\prime}$ and such that

$$
|\Delta \phi|=\left|\phi\left[\tau+\Delta \tau, \tau_{0}+\Delta \tau_{0}, x_{0}+\Delta x_{0}, f+\Delta f\right]-\phi\left[\tau, \tau_{0}, x_{0}, f\right]\right|<\epsilon
$$

whenever the two sets $\left[\tau+\Delta \tau, \tau_{0}+\Delta \tau_{0}, x_{0}+\Delta x_{0}, f+\Delta f\right]$ and $\left[\tau, \tau_{0}\right.$, $\left.x_{0}, f\right]$ are in $R$ and the inequalities

$$
|\Delta \tau|,\left|\Delta \tau_{0}\right|, \bmod \Delta x_{0}, \bmod \Delta f, \bmod \Delta f_{x}<\delta^{\prime},
$$

are true. The last two are understood as holding uniformly for all arguments $(\tau, x)$ of the elements of $\Delta f$ and $\Delta f_{x}$ in $C_{x}$. In order that $\Delta \phi$ shall approach zero, therefore, it is necessary according to this definition, that the derivatives of the elements of $\Delta f$ with respect to the elements of $x$ shall approach zero as well as the elements of $\Delta \tau, \Delta \tau_{0}, \Delta x_{0}, \Delta f$. Furthermore the uniformity of the continuity is with respect to the arguments $\tau, \tau_{0}, x_{0}$ but not with respect to $f$, as.is indicated by the fact that the constant $\delta^{\prime}$ depends upon $f$ as well as upon $\epsilon^{\prime}$.

It is clear that $f_{x}(\tau, y)$ is continuous in the sense just described. For let $\bar{f}=f+\Delta f$, and let $\Delta v$ stand for the difference Then

$$
\Delta v=v\left[\tau+\Delta \tau, \tau_{0}+\Delta \tau_{0}, x_{0}+\Delta x_{0}, f+\Delta f\right]-v\left[\tau, \tau_{0}, x_{0}, f\right]
$$

$$
\begin{aligned}
\bmod \left[\bar{f}_{x}(\tau+\Delta \tau, v+\Delta v)\right. & \left.-f_{x}(\tau, v)\right] \\
\leqq & \bmod \left[\bar{f}_{x}(\tau+\Delta \tau, v+\Delta v)-f_{x}(\tau+\Delta \tau, v+\Delta v)\right] \\
& +\bmod \left[f_{x}(\tau+\Delta \tau, v+\Delta v)-f_{x}(\tau, v)\right] \\
\leqq & \bmod \Delta f_{x}(\tau+\Delta \tau, v+\Delta v) \\
& +\bmod \left[f_{x}(\tau+\Delta \tau, v+\Delta v)-f_{x}(\tau, v)\right]
\end{aligned}
$$


The next to last term on the right approaches zero with $\bmod \Delta f_{x}$, and the last term does the same with the first four of the quantities (8), since the elements of $f_{x}$ and $v$ are continuous. But the uniformity of the approach to zero in the case of the last modulus is with respect to $\tau, \tau_{0}, x_{0}$ only, since a $\delta^{\prime}$ suitable for making that modulus less than $\epsilon^{\prime}$ will in general depend upon $f$. It is easily provable that

$$
\int_{\tau_{0}}^{\tau} f_{x} d \tau, \quad f_{x} \int_{\tau_{0}}^{\tau} f_{x} d \tau
$$

are continuous in the same way, and so by induction that each term of the series (6) has this property. Equations (7) with the equations

$$
\frac{\partial v}{\partial \tau}=f(\tau, v)
$$

now show that all the elements of the first derivatives of both $v$ and $\partial v / \partial \tau$ have continuity of type II. These results may be summarized in the following theorem:

Theorem. Let the arc

$$
x=u(\tau)
$$

$$
\left(\tau_{1} \leqq \tau \leqq \tau_{2}\right)
$$

be a solution of the differential equations

$$
\frac{d x}{d \tau}=f^{\prime}(\tau, x),
$$

where the functions $f^{\prime}(\tau, x)$ are continuous and satisfy the Lipschitz conditions

$$
\bmod \left[f^{\prime}\left(\tau, x^{\prime}\right)-f^{\prime}(\tau, x)\right]<\kappa \bmod \left(x^{\prime}-x\right)
$$

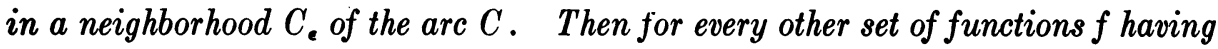
these properties in $C_{e}$ with the same constant $\kappa$ there exists a unique set of solutions

$$
x=v\left[\tau, \tau_{0}, x_{0}, f\right]
$$

through each initial point $\left(\tau_{0}, x_{0}\right)$ sufficiently neár to $C$. The functionals $v\left[\tau, \tau_{0}, x_{0}, f\right]$ so defined are single valued and have uniform continuity of the type I described above in the domain $R$ of sets $\left[\tau, \tau_{0}, x_{0}, f\right]$ defined by the conditions

$$
(R) \tau_{1} \leqq \tau \leqq \tau_{2}, \quad\left(\tau_{0}, x_{0}\right) \text { in } C_{\delta / 2}, \quad \bmod \left(f^{\prime}-f\right) \leqq \frac{\delta}{2\left(\tau_{2}-\tau_{1}\right)} .
$$

If the class of functions $f$ considered is restricted to include only those with continuous first derivatives whose absolute values have a common upper bound in the neighborhood $C_{\mathrm{e}}$, then the functionals (10) and their derivatives $\partial v / \partial \tau$ have partial derivatives with respect to the elements of $\tau, \tau_{0}, x_{0}$ which possess continuity of type II in the domain $R$. 
The results which have been proved above can be extended immediately to the case where the functions $f$ contain a set of parameters $a=\left(a_{1}, \cdots, a_{q}\right)$. The class of functions $f(\tau, x, a)$ now to be considered is the totality of those which are continuous and satisfy the Lipschitz condition (9) with a fixed $\kappa$ for sets of arguments $(\tau, x, a)$ such that $(\tau, x)$ is in $C_{\mathrm{e}}$ and $a$ in a region $\mathfrak{A}$ of points $\left(a_{1}, \cdots, a_{q}\right)$. The proofs already given show that the approximations sets $v_{m}\left[\tau, \tau_{0}, x_{0}, f, a\right]$ aie uniformly continuous and converge uniformly in a region $R_{1}$ of sets $\left[\tau, \tau_{0}, x_{0}, f, a\right]$ satisfying the conditions

$$
\left[\tau, \tau_{0}, x_{0}, f\right] \text { is in } R, a \text { in } \mathfrak{A},
$$

and the limit function $v\left[\tau, \tau_{0}, x_{0}, f, a\right]$ has uniform continuity of type I in $R_{1}$. The conclusions of the theorem hold true in each case when $R$ is replaced by $R_{1}$.

Consider as a special case the class of functions $f$ which are linear in $x$ of the form $f=A x$, where $A$ is a matrix whose elements are functions of $\tau$ and parameters $a=\left(a_{1}, \cdots, a_{q}\right)$. Let the elements of $A$ be restricted to be continuous and to be such that $\bmod A<\kappa$ for all arguments $(\tau, a)$ with $\tau_{1} \leqq \tau \leqq \tau_{2}$ and $a$ in a region $\mathfrak{A}$. Then the Lipschitz condition

$$
\begin{aligned}
\bmod \left[f\left(\tau, x^{\prime}\right)-f(\tau, x)\right]=\bmod A\left(x^{\prime}-x\right) & \\
& \leqq \bmod A \bmod \left(x^{\prime}-x\right) \leqq \kappa \bmod \left(x^{\prime}-x\right)
\end{aligned}
$$

is satisfied for all these functions and they are all continuous in every neighborhood $C_{e}$ of the special arc

$$
x=0
$$$$
\left(\tau_{1} \leqq \tau \leqq \tau_{2}\right)
$$

satisfying the equations (3) with the functions $f^{\prime}(\tau, x) \equiv 0$ substituted. For the system (3) with such sets of functions $f=A x$ the proofs of the preceding paragraphs establish the existence and continuity of the solutions $v\left[\tau, \tau_{0}, x_{0}, A, a\right]$ in a region $R_{2}$ of elements $\left[\tau, \tau_{0}, x_{0}, A, a\right]$ satisfying the conditions

$$
\tau_{1} \leqq \tau \leqq \tau_{2}, \quad \tau_{1} \leqq \tau_{0} \leqq \tau_{2}, \quad \bmod x_{0} \leqq \delta,
$$

$A$ arbitrary except for the restrictions just specified, $a$ in $\mathfrak{A}$.

The constant $\delta$ is in this case entirely arbitrary, for it was originally restricted in order to make the points $\left(\tau, v_{m}\right)$ lie in the neighborhood $C_{a}$ where the properties of the functions $f$ were presupposed, and when the elements of $f$ are linear in $x$ this neighborhood is $C_{\infty}$. The proof that

$$
\bmod \left(v_{m}-u\right)=\bmod v_{m} \leqq \delta e^{\kappa \lambda}
$$

is, however, still valid, so that the points $\left(\tau_{1}, v_{m}\right)$ all lie in the neighborhood $C$. for which $\epsilon=\delta e^{\kappa \lambda}$. The continuity of $x\left[\tau, \tau_{0}, x_{0}, A, a\right]$ in this case is such that for every $\epsilon^{\prime}$ there exists a $\delta^{\prime}$ such that 
$\bmod \left(x\left[\tau+\Delta \tau, \tau_{0}+\Delta \tau_{0}, x_{0}+\Delta x_{0}, A+\Delta A, a+\Delta a\right]\right.$

$$
\left.-x\left[\tau, \tau_{0}, x_{0}, A, a\right]\right)<\epsilon^{\prime}
$$

whenever the two sets of arguments of $x$ are in $R_{2}$ and

$$
|\Delta \tau|,\left|\Delta \tau_{0}\right|, \bmod \Delta x_{0}, \bmod \Delta A, \bmod \Delta a<\delta^{\prime} .
$$

\section{Difference functions and Differentials of the solutions}

Under the assumptions made in the last paragraph of the theorem of the preceding section the functionals $x\left[\tau, \tau_{0}, x_{0}, f\right]$ have not only continuous derivatives with respect to the arguments $\tau, \tau_{0}, x_{0}$ but also differentials with respect to the arguments $f$ in a sense analogous to that of the theory of functions of lines. As a first step toward the proof of this statement it is to be shown that for every pair of sets of elements $\left[\tau, \tau_{0}, x_{0}, f\right],\left[\tau, \tau_{0}, x_{0}, \bar{f}\right]$ of the region $R$ there exists a matrix of functions $L\left[\tau^{\prime}, \tau, \tau_{0}, x_{0}, f, \bar{f}\right]$ such that the differences

$$
\Delta v=v\left[\tau, \tau_{0}, x_{0}, \bar{f}\right]-v\left[\tau, \tau_{0}, x_{0}, f\right]
$$

are expressible in the form

$$
\Delta v=\int_{\tau_{0}}^{\tau} L\left[\tau^{\prime}, \tau, \tau_{0}, x_{0}, f, \bar{f}\right] \Delta f\left(\tau^{\prime}, v\left[\tau^{\prime}, \tau_{0}, x_{0}, f\right]\right) d \tau^{\prime},
$$

where $\Delta f=\bar{f}-f$. The elements of $L$ have continuity of type II for all arguments $\left[\tau^{\prime}, \tau, \tau_{0}, x_{0}, f, \bar{f}\right]$ satisfying the conditions

$$
\tau_{1} \leqq \tau^{\prime} \leqq \tau_{2}, \quad\left[{ }^{\prime} \tau, \tau_{0}, x_{0}, f\right] \text { and }\left[\tau, \tau_{0}, x_{0}, \bar{f}\right] \text { in } R
$$

An application of Taylor's theorem with integral form of remainder term* shows that the differences $\Delta v$ satisfy the differential equations

$$
\begin{aligned}
\frac{d}{d \tau} \Delta v & =\bar{f}(\tau, v+\Delta v)-f(\tau, v) \\
& =\bar{f}(\tau, v+\Delta v)-\bar{f}(\tau, v)+\Delta f(\tau, v) \\
& =A \Delta v+\Delta f(\tau, v),
\end{aligned}
$$

where $A$ is the matrix of elements

$$
A=\int_{0}^{1} \bar{f}_{x}(\tau, v+\alpha \Delta v) d \alpha .
$$

The second members of the associated set of differential equations

$$
\frac{d l}{d \tau}=-l A=g\left(\tau, l, \tau_{0}, x_{0}\right)
$$

* See, for example, Jordan, Cours d'analyse, vol. 1, p. 247. 
are linear in the elements of $l$, and the elements of the matrix $A$ are functions of $\tau$ and the variable parameters $\tau_{0}, x_{0}$. Furthermore for each fixed pair $f, \bar{f}$ the elements of $A$ are continuous and such that $\bmod A \leqq \kappa$ in the domain of elements satisfying the conditions

$$
\tau_{1} \leqq \tau \leqq \tau_{2}, \quad\left(\tau_{0}, x_{0}\right) \text { in } C_{\delta / 2} .
$$

The equations (15) are therefore of the form considered in the last paragraph of Section 1 with $a$ replaced by $\tau_{0}, x_{0}$, and $\mathfrak{A}$ by $C_{\delta / 2}$. Hence they have a matrix $L\left[\tau, \tau^{\prime}, A, \tau_{0}, x_{0}\right]$ whose rows are solutions of the equations (15), reducing to the identity matrix $I$ for the special values $\tau=\tau^{\prime}$, and having continuity of type $\mathrm{I}$ for all sets $\left[\tau, \tau^{\prime}, A, \tau_{0}, x_{0}\right]$ satisfying the conditions

$$
\tau_{1} \leqq \tau \leqq \tau_{2}, \quad \tau_{1} \leqq \tau^{\prime} \leqq \tau_{2}, \quad A \text { arbitrary, } \quad\left(\tau_{0}, x_{0}\right) \text { in } C_{\delta / 2} .
$$

Since in the particular case when $A$ has the value (14) its elements have continuity of type II in the domain of elements satisfying the conditions (12), it follows that $L$ may be regarded as having the form $L\left[\tau^{\prime}, \tau, \tau_{0}, x_{0}, f, \bar{f}\right]$ with continuity of type II in the same domain.

If the two members of the equation (13) are multiplied by the matrix $L$ it follows with the help of equation (15) that

$$
L \frac{d}{d \tau} \Delta v=-\frac{d L}{d \tau} \Delta v+L \Delta f(\tau,, v),
$$

and integration of this equation from $\tau_{0}$ to $\tau^{\prime}$.gives

$$
[\Delta v]^{\tau=\tau^{\prime}}=\int_{\tau_{0}}^{\tau^{\prime}} L\left[\tau, \tau^{\prime}, \tau_{0}, x_{0}, f, \bar{f}\right] \Delta f(\tau, v) d \tau
$$

since at $\tau=\tau^{\prime}, L=I$, and at $\tau=\tau_{0}, \Delta v=0$. If the variables $\tau^{\prime}$ and $\tau$ are interchanged in this equation it takes exactly the form (11) which was to be deduced.

The expression (11) is called a difference function of $v\left[\tau, \tau_{0}, x_{0}, f\right]$ with respect to $f$. It is easy to see that $v$ has a difference function of a similar sort with respect to all of its arguments. For let

$$
\begin{aligned}
\Delta_{1} v & =v\left[\bar{\tau}, \bar{\tau}_{0}, \bar{x}_{0}, \bar{f}\right]-v\left[\tau, \tau_{0}, x_{0}, \bar{f}\right], \\
\Delta_{2} v & =v\left[\tau, \tau_{0}, x_{0}, \bar{f}\right]-v\left[\tau, \tau_{0}, x_{0}, f\right], \\
\Delta v & =\Delta_{1} v+\Delta_{2} v,
\end{aligned}
$$

and let $\Delta \tau=\tau-\tau$, with similar interpretations for $\Delta \tau_{0}, \Delta x_{0}, \Delta f$. Then since the functions $v$ have continuous partial derivatives with respect to $\tau$, $\tau_{0}, x_{0}$ it follows that by an application of Taylor's formula with integral form of the remainder term to $\Delta_{1} v$, and an application of formula (11) to $\Delta_{2} v$, that 
where

$$
\Delta v=a \Delta \tau+b \Delta \tau_{0}+C \Delta x_{0}+\int_{\tau_{0}}^{\tau} L \Delta f d \tau^{\prime}
$$

$$
a=\int_{0}^{\cdot} v_{\tau} d \alpha, \quad b=\int_{0}^{1} v_{\tau_{0}} d \alpha, \quad C=\int_{0}^{1} v_{x_{0}} d \alpha,
$$

the arguments of the derivatives $v_{\tau}, v_{\tau_{0}}, v_{x_{0}}$ being $\tau+\alpha \Delta \tau, \tau_{0}+\alpha \Delta \tau_{0}$, $x_{0}+\alpha \Delta x_{0}, \bar{f}$. The elements of $a, b, C$ have clearly continuity of type II for all elements $\left[\bar{\tau}, \bar{\tau}_{0}, \bar{x}_{0}, \bar{f}\right]$ and $\left[\tau, \tau_{0}, x_{0}, f\right]$ in $R$ since the derivatives of $v$ have this property.

The difference

$$
\Delta v=v\left[\tau, \bar{\tau}_{0}, \bar{x}_{0}, \bar{f}\right]-v\left[\tau, \tau_{0}, x_{0}, f\right]
$$

is expressible in the form (16), in which $a, b, C, L$ have elements which are functions of $\tau^{\prime}$ and the elements of the sets $\left[\bar{\tau}, \bar{\tau}_{0}, \bar{x}_{0}, \bar{f}\right]$ and $\left[\tau, \tau_{0}, x_{0}, f\right]$, and which have continuity of type II when these variables satisfy the conditions (12).

It is interesting to note that when the functions $v$ have difference functions of the type (16), they will also have differentials in a sense analogous to that defined by Fréchet in the theory of functions of lines. In the first place, when $\bar{\tau}=\tau, \bar{\tau}_{0}=\tau_{0}, \bar{x}_{0}=x_{0}, \bar{f}=f$, the values of the expressions (17) are simply $a=v_{\tau}, b=v_{\tau_{0}}, C=v_{x_{0}}$. Furthermore if $L^{\prime}$ denotes the value of $L$ for the same arguments, and if $\delta v$ is used to represent the expression

$$
\delta v=v_{\tau} \Delta \tau+v_{\tau_{0}} \Delta \tau_{0}+v_{x_{0}} \Delta x_{0}+\int_{\tau_{0}}^{\tau} L^{\prime} \Delta f d \tau^{\prime}
$$

then it follows with the help of equation (16) that

$$
\Delta v=\delta v+\mu h\left[\Delta \tau, \Delta \tau_{0}, \Delta x_{0}, \Delta f\right]
$$

where $\mu$ is the maximum of $|\Delta \tau|,\left|\Delta \tau_{0}\right|, \bmod \Delta x_{0}, \bmod \Delta f, \bmod \Delta f_{x}$ in $C_{\epsilon}$, and $h$ is a multi-partite function whose modulus approaches zero with $\mu$. For the value of $h$ is in fact

$$
h=\left(a-v_{\tau}\right) \frac{\Delta \tau}{\mu}+\left(b-v_{\tau_{0}}\right) \frac{\Delta \tau_{0}}{\mu}+\left(C-v_{x_{0}}\right) \frac{\Delta x_{0}}{\mu}+\int_{\tau_{0}}^{\tau}\left(L-L^{\prime}\right) \frac{\Delta f}{\mu} d \tau^{\prime},
$$

and the modulus of this approaches zero with $\mu$ since $|\Delta \tau / \mu|,\left|\Delta \tau_{0} / \mu\right|$, $\bmod \left(\Delta x_{0} / \mu\right), \bmod (\Delta f / \mu)$ are at most equal to unity, and $a, b, C, L$ approach continuously the limiting values $v_{\tau}, v_{\tau_{0}}, v_{x_{0}}, L^{\prime}$ when the elements of $\left[\bar{\tau}, \bar{\tau}_{0}\right.$, $\left.\bar{x}_{0}, \bar{f}, \bar{f}_{x}\right]$ approach those of $\left[\tau, \tau_{0}, x_{0}, f, f_{x}\right]$.

The expression (18) is called the differential of $v$ at the set $\left[\tau, \tau_{0}, x_{0}, f\right]$. It gives the value of $v$ to terms of the first order in the variables $\Delta \tau, \Delta \tau_{0}, \Delta x_{0}, \Delta f$ as described above. The matrix $L^{\prime}$ is the matrix whose rows are solutions of the differential equations 


$$
d l=-l f_{x}\left(\tau^{\prime}, v\left[\tau^{\prime}, \tau_{0}, x_{0}, f\right]\right)
$$

with the initial values $L=I$ at $\tau^{\prime}=\tau$.

The last statement of this theorem is true because the second members of equations (15) reduce to those of (20) when $\left[\bar{\tau}, \bar{\tau}_{0}, \bar{x}_{0}, \bar{f}\right]$ and $\left.\tau_{0}, x_{0}, f\right]$ coincide.

It is useful to note that the differences $\Delta v$ are infinitesimals of the first order at least with respect to the variable $\mu$, and that the quotients $\Delta v / \mu$ remain finite as $\mu$ approaches zero. This follows readily by dividing through the expression (16) for $\Delta v$ by $\mu$ and remembering that the quotients $\Delta \tau / \mu$, $\Delta \tau_{0} / \mu, \Delta x_{0} / \mu, \Delta f / \mu$ are all bounded as $\mu$ approaches zero.

\section{ThEOREMS CONCERNING IMPLICIT FUNCTIONS}

Consider an equation of the form

$$
\phi\left[\tau, \tau_{0}, x_{0}, f\right]=0
$$

having an initial solution $\left[\tau^{\prime}, \tau_{0}^{\prime}, x_{0}^{\prime}, f^{\prime}\right]$, and let $\left[\tau^{\prime}, \tau_{0}^{\prime}, x_{0}^{\prime}, f^{\prime}\right]_{\text {e denote the }}$ totality of elements $\left[\tau, \tau_{0}, x_{0}, f\right]$ such that

$$
\left|\tau-\tau^{\prime}\right|,\left|\tau_{0}-\tau_{0}^{\prime}\right|, \bmod \left(x_{0}-x_{0}^{\prime}\right), \bmod \left(f-f^{\prime}\right) \leqq \epsilon .
$$

Then it is possible to prove the following theorem:

Theorem. The function $\phi$ and its derivative $\phi_{\tau}$ are supposed to have continuity of type $I$ in a neighborhood $\left[\tau^{\prime}, \tau_{0}^{\prime}, x_{0}^{\prime}, f^{\prime}\right]_{e}$, and it is furthermore presupposed that

$$
\phi\left[\tau^{\prime}, \tau_{0}^{\prime}, x_{0}^{\prime}, f^{\prime}\right]=0, \quad \phi_{\tau}\left[\tau^{\prime}, \tau_{0}^{\prime}, x_{0}^{\prime}, f^{\prime}\right] \neq 0 .
$$

Then it will be true that

(1) the constant $\epsilon$ can be so restricted that in the neighborhood $\left[\tau^{\prime}, \tau_{0}^{\prime}, x_{0}^{\prime}, f^{\prime}\right]_{\text {e }}$ no two solutions $\left[\tau_{1}, \tau_{0}, x_{0}, f\right],\left[\tau_{2}, \tau_{0}, x_{0}, f\right]$ of the equation $\phi=0$ exist with $\tau_{1} \neq \tau_{2}$;

(2) there is a neighborhood $\left[\tau_{0}^{\prime}, x_{0}^{\prime}, f^{\prime}\right]_{\delta}$ with every element of which there is associated a solution $\left[\tau, \tau_{0}, x_{0}, f\right]$ in $\left[\tau^{\prime}, \tau_{0}^{\prime}, x_{0}^{\prime}, f^{\prime}\right]_{e}$;

(3) the function $\tau\left[\tau_{0}, x_{0}, f\right]$ so defined has continuity of type $I$ in $\left[\tau_{0}^{\prime}, x_{0}^{\prime}, f^{\prime}\right]_{\delta}$.

It is an immediate consequence of the theorem that $\tau^{\prime}=\tau\left[\tau_{0}^{\prime}, x_{0}^{\prime}, f^{\prime}\right]$.

To prove the theorem it may first be remarked that on account of the continuity of the derivative $\phi_{\tau}$ the neighborhood $\left[\tau^{\prime}, \tau_{0}^{\prime}, x_{0}^{\prime}, f^{\prime}\right]_{\text {e can }}$ be so restricted that $\phi_{\tau} \neq 0$ everywhere in it. The conclusion (1) of the theorem is justified in this neighborhood since $\phi$ is there a monotonic function of $\tau$ vanishing at most once in the interval $\left|\tau-\tau^{\prime}\right| \leqq \epsilon$ when $\tau_{0}, x_{0}, f$ are fixed.

The values $\phi\left[\tau^{\prime}+\epsilon, \tau_{0}^{\prime}, x_{0}^{\prime}, f^{\prime}\right]$ and $\phi\left[\tau^{\prime}-\epsilon, \tau_{0}^{\prime}, x_{0}^{\prime}, f^{\prime}\right]$ have now opposite signs, and on account of the continuity of $\phi$ it is possible to choose $\delta \leqq \epsilon$ 
so small that $\phi\left[\tau^{\prime}+\epsilon, \tau_{0}, \xi_{0}, f\right]$ and $\phi\left[\tau^{\prime}-\epsilon, \tau_{0}, x_{0}, f\right]$ will also have opposite signs for every $\left[\tau_{0}, x_{0}, f\right]$ in the neighborhood $\left[\tau_{0}^{\prime}, x_{0}^{\prime}, f^{\prime}\right]_{\delta}$. For every such $\left[\tau_{0}, x_{0}, f\right]$ the function $\phi\left[\tau, \tau_{0}, x_{0}, f\right]$ will be a monotonic function of $\tau$ and will vanish at one value on the interval $\tau^{\prime}-\epsilon \leqq \tau \leqq \tau^{\prime}+\epsilon$, which proves the conclusion (2) of the theorem.

For two solutions $\left[\tau, \tau_{0}, x_{0}, f\right],\left[\tau+\Delta \tau, \tau_{0}+\Delta \tau_{0}, x_{0}+\Delta x_{0}, f+\Delta f\right]$ with projections in the neighborhood. $\left[\tau_{0}^{\prime}, x_{0}^{\prime}, f^{\prime}\right]_{\delta}$ the equations

$$
\begin{aligned}
0 & =\phi\left[\tau+\Delta \tau, \tau_{0}+\Delta \tau_{0}, x_{0}+\Delta x_{0}, f+\Delta f\right] \\
& -\phi\left[\tau, \tau_{0}+\Delta \tau_{0}, x_{0}+\Delta x_{0}, f+\Delta f\right] \\
+\phi\left[\tau, \tau_{0}+\Delta \tau_{0}, x_{0}+\Delta x_{0}, f+\Delta f\right] & -\phi\left[\tau, \tau_{0}, x_{0}, f\right] \\
& =\phi_{1} \Delta \tau+\phi\left[\tau, \tau_{0}+\Delta \tau_{0}, x_{0}+\Delta x_{0}, f+\Delta f\right]-\phi\left[\tau, \tau_{0}, x_{0}, f\right]
\end{aligned}
$$

are true, where

$$
\phi_{1}=\int_{0}^{1} \phi_{\tau}\left[\tau+\alpha \Delta \tau, \tau_{0}+\Delta \tau_{0}, x_{0}+\Delta x_{0}, f+\Delta f\right] d \alpha .
$$

Since $\phi$ is continuous and $\phi_{1} \neq 0$ it follows that $\Delta \tau$ approaches zero with $\left|\Delta \tau_{0}\right|, \bmod \Delta x_{0}, \bmod \Delta f$, and hence that the function $\tau\left[\tau_{0}, x_{0}, f\right]$ is continuous in $\left[\tau_{0}^{\prime}, x_{0}^{\prime}, f^{\prime}\right]_{\delta}$, as stated in conclusion (3) of the theorem.

If the function $\phi$ is one of the functions $v$ of the preceding section, the solution $\tau\left[\tau_{0}, x_{0}, f\right]$ of the equation $\phi=0$ will have a difference function

$$
\Delta \tau=-\frac{\beta}{\alpha} \Delta \tau_{0}-\frac{c}{\alpha} \Delta x_{0}-\frac{1}{\alpha} \int_{\tau_{0}}^{\tau} l \Delta f d \tau^{\prime},
$$

where $\alpha, \beta, c, l$ represent those of the elements of $a b, C, L$ in equation (16) which pertain to the particular function $\phi$. In them the arguments $\tau, \bar{\tau}$ are supposed replaced by the functions $\tau\left[\tau_{0}, x_{0}, f\right], \tau\left[\bar{\tau}_{0}, \bar{x}_{0}, \bar{f}\right]$.

This is readily provable because the equation

$$
\Delta \phi=\alpha \Delta \tau+\beta \Delta \tau_{0}+c \Delta x_{0}+\int_{\tau_{0}}^{\tau} l \Delta f d \tau^{\prime}
$$

holds for arbitrary arguments $\left[\tau, \tau_{0}, x_{0}, f\right],\left[\bar{\tau}, \bar{\tau}_{0}, \bar{x}_{0}, \bar{f}\right]$, and these sets make both members of the equation vanish when $\tau=\tau\left[\tau_{0}, x_{0}, f\right]$ and $\bar{\tau}=\tau\left[\bar{\tau}_{0}, \bar{x}_{0}, \bar{f}\right]$, since then both of the sets are solutions of the equation $\phi=0$.

If $\phi$ and $\psi$ are both particular functions of the set $v$ of the preceding sections, and if the solution $\tau\left[\tau_{0}, x_{0}, f\right]$ of the equation $\phi=0$ is substituted in $\psi$, then the resulting function $\psi$ of $\tau_{0}, x_{0}, f$ has a difference function 


$$
\begin{aligned}
\Delta_{1} \psi= & -\frac{\bar{\alpha}}{\alpha}\left(\beta \Delta \tau_{0}+c \Delta x_{0}+\int_{\tau_{0}}^{\tau} l \Delta f d \tau^{\prime}\right) \\
& +\bar{\beta} \Delta \tau_{0}+\bar{c} \Delta x_{0}+\int_{\tau_{0}}^{\tau} \bar{l} \Delta f d \tau^{\prime},
\end{aligned}
$$

where $\bar{\alpha}, \bar{\beta}, \bar{c}, \bar{l}$ are symbols for those elements of $a, b, C, L$ in equations (16) which belong to $\psi$, with the arguments $\tau=\tau\left[\tau_{0}, x_{0}, f\right], \bar{\tau}=\tau\left[\bar{\tau}_{0}, \bar{x}_{0}, \bar{f}\right]$.

This result is found by substituting the expression (21) for $\Delta \tau$ in the difference function

$$
\Delta \psi=\bar{\alpha} \Delta \tau+\bar{\beta} \Delta \tau_{0}+\bar{c} \Delta x_{0}+\int_{\tau_{0}}^{\tau} \bar{l} \Delta f d \tau^{\prime}
$$

of the function $\psi$. The differential $\delta_{1} \psi$ of $\psi$ with respect to the arguments $\tau_{0}, x_{0}, f$ after the substitution $\tau=\tau\left[\tau_{0}, x_{0}, f\right]$ is evidently

$$
\begin{aligned}
\delta_{1} \psi= & -\frac{\psi_{\tau}}{\phi_{\tau}}\left(\phi_{\tau_{0}} \Delta \tau_{0}+\phi_{x_{0}} \Delta x_{0}+\int_{\tau_{0}}^{\tau} l^{\prime} \Delta f d \tau^{\prime}\right) \\
& +\psi_{\tau_{0}} \Delta \tau_{0}+\psi_{x_{0}} \Delta x_{0}+\int_{\tau_{0}}^{\tau} \overline{l^{\prime}} \Delta f d \tau^{\prime},
\end{aligned}
$$

where the arguments of the derivatives of $\phi, \psi$ are $\tau\left[\tau_{0}, x_{0}, f\right], \tau_{0}, x_{0}, f$. 